\title{
Simple, Robust and (almost) Unsupervised Generation of Polarity Lexicons for Multiple Languages
}

\author{
Iñaki San Vicente, Rodrigo Agerri, German Rigau \\ IXA NLP Group \\ University of the Basque Country (UPV/EHU) \\ Donostia-San Sebastián \\ \{inaki.sanvicente, rodrigo.agerri,german.rigau\}@ehu.es
}

\begin{abstract}
This paper presents a simple, robust and (almost) unsupervised dictionary-based method, qwn-ppv (Q-WordNet as Personalized PageRanking Vector) to automatically generate polarity lexicons. We show that qwn-ppv outperforms other automatically generated lexicons for the four extrinsic evaluations presented here. It also shows very competitive and robust results with respect to manually annotated ones. Results suggest that no single lexicon is best for every task and dataset and that the intrinsic evaluation of polarity lexicons is not a good performance indicator on a Sentiment Analysis task. The qwn-ppv method allows to easily create quality polarity lexicons whenever no domain-based annotated corpora are available for a given language.
\end{abstract}

\section{Introduction}

Opinion Mining and Sentiment Analysis are important for determining opinions about commercial products, on companies reputation management, brand monitoring, or to track attitudes by mining social media, etc. Given the explosion of information produced and shared via the Internet, it is not possible to keep up with the constant flow of new information by manual methods.

Sentiment Analysis often relies on the availability of words and phrases annotated according to the positive or negative connotations they convey. 'Beautiful', 'wonderful', and 'amazing' are examples of positive words whereas 'bad', 'awful', and 'poor' are examples of negatives.

The creation of lists of sentiment words has generally been performed by means of manual-, dictionary- and corpus-based methods. Manually collecting such lists of polarity annotated words is labor intensive and time consuming, and is thus usually combined with automated approaches as the final check to correct mistakes. However, there are well known lexicons which have been fully (Stone et al., 1966; Taboada et al., 2010) or at least partially manually created (Hu and Liu, 2004; Riloff and Wiebe, 2003).

Dictionary-based methods rely on some dictionary or lexical knowledge base (LKB) such as WordNet (Fellbaum and Miller, 1998) that contain synonyms and antonyms for each word. A simple technique in this approach is to start with some sentiment words as seeds which are then used to perform some iterative propagation on the LKB (Hu and Liu, 2004; Strapparava and Valitutti, 2004; Kim and Hovy, 2004; Takamura et al., 2005; Turney and Littman, 2003; Mohammad et al., 2009; Agerri and García-Serrano, 2010; Baccianella et al., 2010).

Corpus-based methods have usually been applied to obtain domain-specific polarity lexicons: they have been created by either starting from a seed list of known words and trying to find other related words in a corpus or by attempting to directly adapt a given lexicon to a new one using a domain-specific corpus (Hatzivassiloglou and McKeown, 1997; Turney and Littman, 2003; Ding et al., 2008; Choi and Cardie, 2009; Mihalcea et al., 2007). One particular issue arising from corpus methods is that for a given domain the same word can be positive in one context but negative in another. This is also a problem shared by manual and dictionary-based methods, and that is why $q w n-p p v$ also produces synset-based lexicons for approaches on Sentiment Analysis at sense level.

This paper presents a simple, robust and (almost) unsupervised dictionary-based method, QWordNet-PPV (QWordNet by Personalized PageRank Vector) to automatically generate polarity lexicons based on propagating some automatically created seeds using a Personalized 
PageRank algorithm (Agirre et al., 2014; Agirre and Soroa, 2009) over a LKB projected into a graph. We see $q w n-p p v$ as an effective methodology to easily create polarity lexicons for any language for which a WordNet is available.

This paper empirically shows that: (i) qwn-ppv outperforms other automatically generated lexicons (e.g. SentiWordNet 3.0, MSOL) on the 4 extrinsic evaluations presented here; it also displays competitive and robust results also with respect to manually annotated lexicons; (ii) no single polarity lexicon is fit for every Sentiment Analysis task; depending on the text data and the task itself, one lexicon will perform better than others; (iii) if required, $q w n-p p v$ efficently generates many lexicons on demand, depending on the task on which they will be used; (iv) intrinsic evaluation is not appropriate to judge whether a polarity lexicon is fit for a given Sentiment Analysis (SA) task because good correlation with respect to a gold-standard does not correspond with correlation with respect to a SA task; (v) it is easily applicable to create $q w n-p p v(s)$ for other languages, and we demonstrate it here by creating many polarity lexicons not only for English but also for Spanish; (vi) the method works at both word and sense levels and it only requires the availability of a LKB or dictionary; finally, (vii) a dictionarybased method like qwn-ppv allows to easily create quality polarity lexicons whenever no domainbased annotated reviews are available for a given language. After all, there usually is available a dictionary for a given language; for example, the Open Multilingual WordNet site lists WordNets for up to 57 languages (Bond and Foster, 2013).

Although there has been previous work using graph methods for obtaining lexicons via propagation, the qwn-ppv method to combine the seed generation and the Personalized PageRank propagation is novel. Furthermore, it is considerable simpler and obtains better and easier to reproduce results than previous automatic approaches (Esuli and Sebastiani, 2007; Mohammad et al., 2009; Rao and Ravichandran, 2009).

Next section reviews previous related work, taking special interest on those that are currently available for evaluation purposes. Section $3 \mathrm{de}-$ scribes the qwn-ppv method to automatically generate lexicons. The resulting lexical resources are evaluated in section 4 . We finish with some concluding remarks and future work in section 5 .

\section{Related Work}

There is a large amount of work on Sentiment Analysis and Opinion Mining, and good comprehensive overviews are already available (Pang and Lee, 2008; Liu, 2012), so we will review the most representative and closest to the present work. This means that we will not be reviewing corpus-based approaches but rather those constructed manually or upon a dictionary or LKB. We will in turn use the approaches here reviewed for comparison with qwn-ppv in section 4 .

The most popular manually-built polarity lexicon is part of the General Inquirer (Stone et al., 1966), and consists of 1915 words labelled as "positive" and 2291 as "negative". Taboada et al. (2010) manually created their lexicons annotating the polarity of 6232 words on a scale of 5 to -5 . Liu et al., starting with $\mathrm{Hu}$ and Liu (2004), have along the years collected a manually corrected polarity lexicon which is formed by 4818 negative and 2041 positive words. Another manually corrected lexicon (Riloff and Wiebe, 2003) is the one used by the Opinion Finder system (Wilson et al., 2005) and contains 4903 negatively and 2718 positively annotated words respectively.

Among the automatically built lexicons, Turney and Littman (2003) proposed a minimally supervised algorithm to calculate the polarity of a word depending on whether it co-ocurred more with a previously collected small set of positive words rather than with a set of negative ones. Agerri and García Serrano presented a very simple method to extract the polarity information starting from the quality synset in WordNet (Agerri and GarcíaSerrano, 2010). Mohammad et al. (2009) developed a method in which they first identify (by means of affixes rules) a set of positive/negative words which act as seeds, then used a Roget-like thesaurus to mark the synonymous words for each polarity type and to generalize from the seeds. They produce several lexicons the best of which, MSOL(ASL and GI) contains $51 \mathrm{~K}$ and $76 \mathrm{~K}$ entries respectively and uses the full General Inquirer as seeds. They performed both intrinsic and extrinsic evaluations using the MPQA 1.1 corpus.

Finally, there are two approaches that are somewhat closer to us, because they are based on WordNet and graph-based methods. SentiWordNet 3.0 (Baccianella et al., 2010) is built in 4 steps: (i) they select the synsets of 14 paradigmatic positive and negative words used as seeds (Turney 
and Littman, 2003). These seeds are then iteratively extended following the construction of WordNet-Affect (Strapparava and Valitutti, 2004). (ii) They train 7 supervised classifiers with the synsets' glosses which are used to assign polarity and objectivity scores to WordNet senses. (iii) In SentiWordNet 3.0 (Esuli and Sebastiani, 2007) they take the output of the supervised classifiers as input to applying PageRank to WordNet 3.0's graph. (iv) They intrinsically evaluate it with respect to MicroWnOp-3.0 using the p-normalized Kendall $\tau$ distance (Baccianella et al., 2010). Rao and Ravichandran (2009) apply different semisupervised graph algorithms (Mincuts, Randomized Mincuts and Label Propagation) to a set of seeds constructed from the General Inquirer. They evaluate the generated lexicons intrinsically taking the General Inquirer as the gold standard for those words that had a match in the generated lexicons.

In this paper, we describe two methods to automatically generate seeds either by following Agerri and García-Serrano (2010) or using Turney and Littman's (2003) seeds. The automatically obtained seeds are then fed into a Personalized PageRank algorithm which is applied over a WordNet projected on a graph. This method is fully automatic, simple and unsupervised as it only relies on the availability of a LKB.

\section{Generating qwn-ppv}

The overall procedure of our approach consists of two steps: (1) automatically creates a set of seeds by iterating over a LKB (e.g. a WordNet) relations; and (2) uses the seeds to initialize contexts to propagate over the LKB graph using a Personalized Pagerank algorithm. The result is qwn-ppv(s): Q-WordNets as Personalized PageRanking Vectors.

\subsection{Seed Generation}

We generate seeds by means of two different automatic procedures.

1. AG: We start at the quality synset of WordNet and iterate over WordNet relations following the original Q-WordNet method described in Agerri and García Serrano (2010).

2. TL: We take a short manually created list of 14 positive and negative words (Turney and Littman, 2003) and iterate over WordNet using five relations: antonymy, similarity, derived-from, pertains-to and also-see.

The AG method starts the propagation from the attributes of the quality synset in WordNet. There are five noun quality senses in WordNet, two of which contain attribute relations (to adjectives). From the quality ${ }_{n}^{1}$ synset the attribute relation takes us to positive $e_{a}^{1}$, negative ${ }_{a}^{1}, \operatorname{good}_{a}^{1}$ and bad $_{a}^{1}$; quality $y_{n}^{2}$ leads to the attributes superior ${ }_{a}^{1}$ and inferior ${ }_{a}^{2}$. The following step is to iterate through every WordNet relation collecting (i.e., annotating) those synsets that are accessible from the seeds. Both $A G$ and $T L$ methods to generate seeds rely on a number of relations to obtain a more balanced POS distribution in the output synsets. The output of both methods is a list of (assumed to be) positive and negative synsets. Depending on the number of iterations performed a different number of seeds to feed UKB is obtained. Seed numbers vary from 100 hundred to $10 \mathrm{~K}$ synsets. Both seed creation methods can be applied to any WordNet, not only Princeton WordNet, as we show in section 4.

\subsection{PPV generation}

The second and last step to generate qwn-ppv(s) consists of propagating over a WordNet graph to obtain a Personalized PageRanking Vector (PPV), one for each polarity. This step requires:

1. A LKB projected over a graph.

2. A Personalized PageRanking algorithm which is applied over the graph.

3. Seeds to create contexts to start the propagation, either words or synsets.

Several undirected graphs based on WordNet 3.0 as represented by the MCR 3.0 (Agirre et al., 2012) have been created for the experimentation, which correspond to 4 main sets: (G1) two graphs consisting of every synset linked by the synonymy and antonymy relations; (G2) a graph with the nodes linked by every relation, including glosses; (G3) a graph consisting of the synsets linked by every relation except those that are linked by antonymy; finally, (G4) a graph consisting of the nodes related by every relation except the antonymy and gloss relations.

Using the (G1) graphs, we propagate from the seeds over each type of graph (synonymy and antonymy) to obtain two rankings per polarity. 


\begin{tabular}{|c|c|c|c|c|c|c|c|c|c|c|c|c|c|c|}
\hline \multirow[b]{3}{*}{ Lexicon } & \multicolumn{7}{|c|}{ Synset Level } & \multicolumn{7}{|c|}{ Word level } \\
\hline & \multirow[b]{2}{*}{ size } & \multicolumn{3}{|c|}{ Positives } & \multicolumn{3}{|c|}{ Negatives } & \multirow[b]{2}{*}{ size } & \multicolumn{3}{|c|}{ Positives } & \multicolumn{3}{|c|}{ Negatives } \\
\hline & & $\mathrm{P}$ & $\mathrm{R}$ & $\mathrm{F}$ & $\mathrm{P}$ & $\mathrm{R}$ & $\mathrm{F}$ & & $\mathrm{P}$ & $\mathrm{R}$ & $\mathrm{F}$ & $P$ & $\mathrm{R}$ & $\mathrm{F}$ \\
\hline \multicolumn{15}{|l|}{ Automatically created } \\
\hline MSOL(ASL-GI)* & 32706 & .65 & .45 & .53 & .58 & .76 & .66 & 76400 & .70 & .49 & .58 & .61 & .79 & .69 \\
\hline QWN & 15508 & .69 & .53 & .60 & .62 & .76 & .68 & 11693 & .64 & .53 & .58 & 60 & .70 & .65 \\
\hline SWN & 27854 & .73 & .57 & .64 & .65 & .79 & .71 & 38346 & .70 & .55 & .62 & 63 & .77 & .69 \\
\hline QWN-PPV-AG(s03_G1/w01_G1) & 2589 & .77 & 63 & .69 & .69 & .81 & .74 & 5119 & 68 & 77 & .72 & .73 & .64 & .68 \\
\hline QWN-PPV-TL(s04_G1/w01_G1) & 5010 & .76 & .66 & .70 & .70 & .79 & .74 & 4644 & .68 & .71 & .69 & .70 & .67 & .68 \\
\hline \multicolumn{15}{|l|}{ (Semi-) Manually created } \\
\hline $\mathrm{GI}^{*}$ & 2791 & .74 & .57 & .64 & .65 & .80 & .72 & 3376 & .79 & .64 & .71 & .70 & .83 & .76 \\
\hline $\mathrm{OF}^{*}$ & 4640 & .77 & .61 & .68 & .68 & .81 & .74 & 6860 & .82 & .71 & .76 & .74 & .84 & .79 \\
\hline Liu* & 4127 & .81 & 63 & .71 & .70 & .85 & .76 & 6786 & .85 & .74 & .79 & .77 & .87 & .82 \\
\hline SO-CAL* & 4212 & .75 & .57 & .64 & .65 & .81 & .72 & 6226 & .82 & .70 & .76 & .74 & .85 & .79 \\
\hline
\end{tabular}

Table 1: Evaluation of lexicons at document level using Bespalov's Corpus.

The graphs created in (G2), (G3) and (G4) are used to obtain two ranks, one for each polarity by propagating from the seeds. In all four cases the different polarity rankings have to be combined in order to obtain a final polarity lexicon: the polarity score $\operatorname{pol}(s)$ of a given synset $s$ is computed by adding its scores in the positive rankings and subtracting its scores in the negative rankings. If $\operatorname{pol}(s)>0$ then $s$ is included in the final lexicon as positive. If $\operatorname{pol}(s)<0$ then $s$ is included in the final lexicon as negative. We assume that synsets with null polarity scores have no polarity and consequently they are excluded from the final lexicon.

The Personalized PageRanking propagation is performed starting from both synsets and words and using both $A G$ and $T L$ styles of seed generation, as explained in section 3.1. Combining the various possibilities will produce at least 6 different lexicons for each iteration, depending on which decisions are taken about which graph, seeds and word/synset to create the $q w n-p p v(\mathrm{~s})$. In fact, the experiments produced hundreds of lexicons, according to the different iterations for seed generation ${ }^{1}$, but we will only refer to those that obtain the best results in the extrinsic evaluations.

With respect to the algorithm to propagate over the WordNet graph from the automatically created seeds, we use a Personalized PageRank algorithm (Agirre et al., 2014; Agirre and Soroa, 2009). The famous PageRank (Brin and Page, 1998) algorithm is a method to produce a rank from the vertices in a graph according to their relative structural importance. PageRank has also been viewed as the result of a Random Walk process, where the final rank of a given node represents the probability of a random walk over the graph which ends on that same node. Thus, if we take the created Word-

\footnotetext{
${ }^{1}$ The total time to generate the final 352 QWN-PPV propagations amounted to around two hours of processing time in a standard PC.
}

Net graph $G$ with $N$ vertices $v_{1}, \ldots, v_{n}$ and $d_{i}$ as being the outdegree of node $i$, plus a $N \times N$ transition probability matrix $M$ where $M_{j i}=1 / d_{i}$ if a link from $i$ to $j$ exists and 0 otherwise, then calculating the PageRank vector over a graph $G$ amounts to solve the following equation (1):

$$
\mathbf{P r}=c M \mathbf{P r}+(1-c) \mathbf{v}
$$

In the traditional PageRank, vector $\mathbf{v}$ is a uniform normalized vector whose elements values are all $1 / N$, which means that all nodes in the graph are assigned the same probabilities in case of a random walk. Personalizing the PageRank algorithm in this case means that it is possible to make vector $\mathbf{v}$ non-uniform and assign stronger probabilities to certain nodes, which would make the algorithm to propagate the initial importance of those nodes to their vicinity. Following Agirre et al. (2014), in our approach this translates into initializing vector $\mathbf{v}$ with those senses obtained by the seed generation methods described above in section 3.1. Thus, the initialization of vector $\mathbf{v}$ using the seeds allows the Personalized propagation to assign greater importance to those synsets in the graph identified as being positive and negative, which resuls in a PPV with the weigths skewed towards those nodes initialized/personalized as positive and negative.

\section{Evaluation}

Previous approaches have provided intrinsic evaluation (Mohammad et al., 2009; Rao and Ravichandran, 2009; Baccianella et al., 2010) using manually annotated resources such as the General Inquirer (Stone et al., 1966) as gold standard. To facilitate comparison, we also provide such evaluation in section 4.3. Nevertheless, and as demonstrated by the results of the extrinsic evaluations, we believe that polarity lexicons should 


\begin{tabular}{|c|c|c|c|c|c|c|c|c|c|c|c|c|c|c|}
\hline \multirow[b]{3}{*}{ Lexicon } & \multicolumn{7}{|c|}{ Synset Level } & \multicolumn{7}{|c|}{ Word level } \\
\hline & \multirow[b]{2}{*}{ size } & \multicolumn{3}{|c|}{ Positives } & \multicolumn{3}{|c|}{ Negatives } & \multirow[b]{2}{*}{ size } & \multicolumn{3}{|c|}{ Positives } & \multicolumn{3}{|c|}{ Negatives } \\
\hline & & $\mathrm{P}$ & $\mathrm{R}$ & $\mathrm{F}$ & $\mathrm{P}$ & $\mathrm{R}$ & $\mathrm{F}$ & & $\mathrm{P}$ & $\mathrm{R}$ & $\mathrm{F}$ & $\mathrm{P}$ & $\mathrm{R}$ & $\mathrm{F}$ \\
\hline \multicolumn{15}{|l|}{ Automatically created } \\
\hline MSOL(ASL-GI)* & 32706 & .56 & .37 & .44 & .76 & .87 & .81 & 76400 & .67 & .5 & .57 & .80 & .89 & .85 \\
\hline QWN & 15508 & .63 & .22 & .33 & .73 & .94 & .83 & 11693 & .58 & .22 & .31 & .73 & .93 & .82 \\
\hline SWN & 27854 & .57 & .33 & .42 & .75 & .89 & .81 & 38346 & .55 & .55 & .55 & .80 & .8 & .80 \\
\hline QWN-PPV-AG (w10_G3/s09_G4) & 117485 & .60 & 63 & .62 & .83 & .82 & .83 & 144883 & .65 & .50 & .57 & .80 & .88 & .84 \\
\hline QWN-PPV-TL (s05_G4) & 114698 & .61 & .58 & .59 & .82 & .83 & .83 & 144883 & .66 & .53 & .59 & .81 & .88 & .84 \\
\hline \multicolumn{15}{|l|}{ (Semi-) Manually created } \\
\hline $\mathrm{GI}^{*}$ & 2791 & .70 & .32 & .44 & .76 & .94 & .84 & 3376 & .71 & .56 & .62 & .82 & .90 & .86 \\
\hline OF* $^{*}$ & 4640 & .67 & .37 & .48 & .77 & .92 & .84 & 6860 & .75 & .68 & .71 & .87 & .90 & .88 \\
\hline Liu* & 4127 & .67 & .33 & .44 & .76 & .93 & .83 & 6786 & .78 & .45 & .57 & .79 & .94 & .86 \\
\hline SO-CAL* & 4212 & .69 & .3 & .42 & .75 & .94 & .84 & 6226 & .73 & .53 & .61 & .81 & .91 & .86 \\
\hline
\end{tabular}

Table 2: Evaluation of lexicons using averaged ratio on the MPQA 1.2 $2_{\text {test }}$ Corpus.

in general be evaluated extrinsically. After all, any polarity lexicon is as good as the results obtained by using it for a particular Sentiment Analysis task.

Our goal is to evaluate the polarity lexicons simplifying the evaluation parameters to avoid as many external influences as possible on the results. We compare our work with most of the lexicons reviewed in section 2 , both at synset and word level, both manually and automatically generated: General Inquirer (GI), Opinion Finder (OF), Liu, Taboada et al.'s (SO-CAL), Agerri and García-Serrano (2010) (QWN), Mohammad et al's, (MSOL(ASL-GI)) and SentiWordNet 3.0 (SWN). The results presented in section 4.2 show that extrinsic evaluation is more meaningful to determine the adequacy of a polarity lexicon for a specific Sentiment Analysis task.

\subsection{Datasets and Evaluation System}

Three different corpora were used: Bespalov et al.'s (2011) and MPQA (Riloff and Wiebe, 2003) for English, and HOpinion ${ }^{2}$ in Spanish. In addition, we divided the corpus into two subsets $(75 \%$ development and $25 \%$ test) for applying our ratio system for the phrase polarity task too. Note that the development set is only used to set up the polarity classification task, and that the generation of $q w n-p p v$ lexicons is unsupervised.

For Spanish we tried to reproduce the English settings with Bespalov's corpus. Thus, both development and test sets were created from the HOpinion corpus. As it contains a much higher proportion of positive reviews, we created also subsets which contain a balanced number of positive and negative reviews to allow for a more meaningful comparison than that of table 6 . Table 3 shows the number of documents per polarity for Bespalov's,

\footnotetext{
${ }^{2}$ http://clic.ub.edu/corpus/hopinion
}

MPQA 1.2 and HOpinion.

\begin{tabular}{|c|c|c|c|}
\hline Corpus & POS docs & NEG docs & Total \\
\hline Bespalov $_{d e v}$ & 23,112 & 23,112 & 46,227 \\
\hline Bespalov $_{\text {test }}$ & 10,557 & 10,557 & 21,115 \\
\hline MPQA $1.2_{d e v}$ & 2,315 & 5,260 & 7,575 \\
\hline MPQA $1.2_{\text {test }}$ & 771 & 1,753 & 2,524 \\
\hline MPQA $1.2_{\text {total }}$ & 3,086 & 7,013 & 10,099 \\
\hline HOpinion_Balanced $_{d e v}$ & 1,582 & 1,582 & 3,164 \\
\hline HOpinion_Balanced ${ }_{\text {tes }}$ & 528 & 528 & 1,056 \\
\hline HOpinion $_{d e v}$ & 9,236 & 1,582 & 10,818 \\
\hline HOpinion $_{\text {test }}$ & 3,120 & 528 & 3,648 \\
\hline
\end{tabular}

Table 3: Number of positive and negative documents in train and test sets.

We report results of 4 extrinsic evaluations or tasks, three of them based on a simple ratio average system, inspired by Turney (2002), and another one based on Mohammad et al. (2009). We first implemented a simple average ratio classifier which computes the average ratio of the polarity words found in document $d$ :

$$
\operatorname{polarity}(d)=\frac{\sum_{w \in d} \operatorname{pol}(w)}{|d|}
$$

where, for each polarity, $\operatorname{pol}(w)$ is 1 if $w$ is included in the polarity lexicon and 0 otherwise. Documents that reach a certain threshold are classified as positive, and otherwise as negative. To setup an evaluation enviroment as fair as possible for every lexicon, the threshold is optimised by maximising accuracy over the development data.

Second, we implemented a phrase polarity task identification as described by Mohammad et al. (2009). Their method consists of: (i) if any of the words in the target phrase is contained in the negative lexicon, then the polarity is negative; (ii) if none of the words are negative, and at least one word is in the positive lexicon, then is positive; (iii) the rest are not tagged.

We chose this very simple polarity estimators because our aim was to minimize the role other 


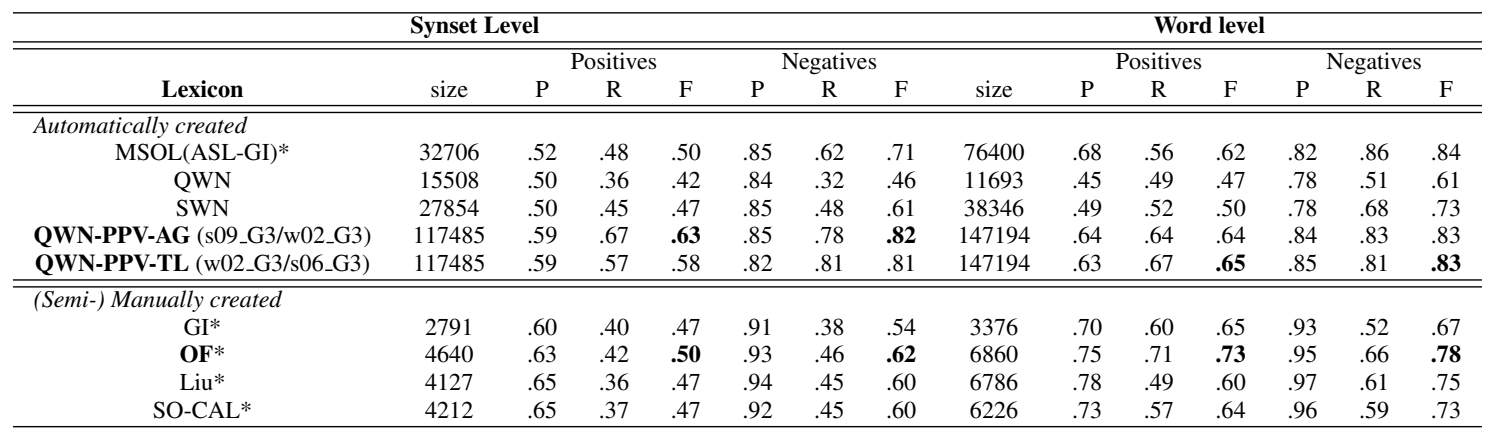

Table 4: Evaluation of lexicons at phrase level using Mohammad et al.'s (2009) method on MPQA $1.2_{\text {total }}$ Corpus.

aspects play in the evaluation and focus on how, other things being equal, polarity lexicons perform in a Sentiment Analysis task. The average ratio is used to present results of tables 1 and 2 (with Bespalov corpus), and 5 and 6 (with HOpinion), whereas Mohammad et al.'s is used to report results in table 4. Mohammad et al.'s (2009) testset based on MPQA 1.1 is smaller, but both MPQA 1.1 and 1.2 are hugely skewed towards negative polarity (30\% positive vs. $70 \%$ negative).

All datasets were POS tagged and Word Sense Disambiguated using FreeLing (Padró and Stanilovsky, 2012; Agirre and Soroa, 2009). Having word sense annotated datasets gives us the opportunity to evaluate the lexicons both at word and sense levels. For the evaluation of those lexicons that are synset-based, such as $q w n-p p v$ and SentiWordNet 3.0, we convert them from senses to words by taking every word or variant contained in each of their senses. Moreover, if a lemma appears as a variant in several synsets the most frequent polarity is assigned to that lemma.

With respect to lexicons at word level, we take the most frequent sense according to WordNet 3.0 for each of their positive and negative words. Note that the latter conversion, for synset based evaluation, is mostly done to show that the evaluation at synset level is harder independently of the quality of the lexicon evaluated.

\subsection{Results}

Although tables 1, 2 and 4 also present results at synset level, it should be noted that the only polarity lexicons available to us for comparison at synset level were Q-WordNet (Agerri and García-Serrano, 2010) and SentiWordNet 3.0 (Baccianella et al., 2010). $Q W N-P P V-A G$ refers to the lexicon generated starting from AG's seeds, and $Q W N-P P V-T L$ using TL's seeds as described in section 3.1. Henceforth, we will use qwn-ppv to refer to the overall method presented in this paper, regardless of the seeds used.

For every qwn-ppv result reported in this section, we have used every graph described in section 3.2. The configuration of each qwn-ppv in the results specifies which seed iteration is used as the initialization of the Personalized PageRank algorithm, and on which graph. Thus, QWN-PPV-TL (s05_G4) in table 2 means that the 5th iteration of synset seeds was used to propagate over graph $\mathrm{G} 4$. If the configuration were (w05_G4) it would have meant 'the 5th iteration of word seeds were used to propagate over graph G4'. The simplicity of our approach allows us to generate many lexicons simply by projecting a LKB over different graphs.

The lexicons marked with an asterisk denote those that have been converted from word to senses using the most frequent sense of WordNet 3.0. We would like to stress again that the purpose of such word to synset conversion is to show that SA tasks at synset level are harder than at word level. In addition, it should also be noted that in the case of SO-CAL (Taboada et al., 2010), we have reduced what is a graded lexicon with scores ranging from 5 to -5 into a binary one.

Table 1 shows that (at least partially) manually built lexicons obtain the best results on this evaluation. It also shows that $q w n-p p v$ clearly outperforms any other automatically built lexicons. Moreover, manually built lexicons suffer from the evaluation at synset level, obtaining most of them lower scores than qwn-ppv, although Liu's (Hu and Liu, 2004) still obtains the best results. In any case, for an unsupervised procedure, qwn-ppv lexicons obtain very competitive results with respect to manually created lexicons and is the best among the automatic methods. It should also be noted that the best results of $q w n-p p v$ are obtained with graph 
G1 and with very few seed iterations.

Table 2 again sees the manually built lexicons performing better although overall the differences are lower with respect to automatically built lexicons. Among these, qwn-ppv again obtains the best results, both at synset and word level, although in the latter the differences with MSOL(ASL-GI) are not large. Finally, table 4 shows that $q w n-p p v$ again outperforms other automatic approaches and is closer to those have been (partially at least) manually built. In both MPQA evaluations the best graph overall to propagate the seeds is G3 because this type of task favours high recall.

\begin{tabular}{lllllllll}
\hline & \multicolumn{4}{c}{ Positives } & \multicolumn{3}{c}{ Negatives } \\
Lexicon & size & $\mathrm{P}$ & $\mathrm{R}$ & $\mathrm{F}$ & $\mathrm{P}$ & $\mathrm{R}$ & $\mathrm{F}$ \\
\hline \hline Automatically created & & & & & & & & \\
SWN & 27854 & .87 & .99 & .93 & .70 & .16 & .27 \\
$\begin{array}{l}\text { QWN-PPV-AG } \\
\text { (wrd01_G1) }\end{array}$ & 3306 & .86 & .00 & .92 & .67 & .01 & .02 \\
$\begin{array}{l}\text { QWN-PPV-TL } \\
\text { (s04_G1) }\end{array}$ & 5010 & .89 & .96 & $\mathbf{. 9 3}$ & .58 & .30 & $\mathbf{. 3 9}$ \\
\hline
\end{tabular}

Table 5: Evaluation of Spanish lexicons using the full HOpinion corpus at synset level.

We report results on the Spanish HOpinion corpus in tables 5 and 6. Mihalcea(f) is a manually revised lexicon based on the automatically built Mihalcea(m) (Pérez-Rosas et al., 2012). ElhPolar (Saralegi and San Vicente, 2013) is semiautomatically built and manually corrected. SOCAL is built manually. SWN and QWN-PPV have been built via the MCR 3.0's ILI by applying the synset to word conversion previously described on the Spanish dictionary of the MCR. The results for Spanish at word level in table 6 show the same trend as for English: qwn-ppv is the best of the automatic approaches and it obtains competitive although not as good as the best of the manually created lexicons (ElhPolar). Due to the disproportionate number of positive reviews, the results for the negative polarity are not useful to draw any meaningful conclusions. Thus, we also performed an evaluation with HOpinion Balanced set as listed in table 3.

The results with a balanced HOpinion, not shown due to lack of space, also confirm the previous trend: $q w n-p p v$ outperforms other automatic approaches but is still worse than the best of the manually created ones (ElhPolar).

\begin{tabular}{lccccccc}
\hline & \multicolumn{4}{c}{ Positives } & \multicolumn{3}{c}{ Negatives } \\
Lexicon & size & P & R & F & P & R & F \\
\hline \hline Automatically created & & & & & & & \\
Mihalcea(m) & 2496 & .86 & .00 & .92 & .00 & .00 & .00 \\
SWN & 9712 & .88 & .97 & .92 & .55 & .19 & .28 \\
QWN-PPV-AG & 1926 & .89 & .97 & .93 & .59 & .26 & .36 \\
(s11_G1) & & & & & & & \\
QWN-PPV-TL & 939 & .89 & .98 &. $\mathbf{9 3}$ & .71 & .26 &. $\mathbf{3 8}$ \\
(s03_G1) & & & & & & & \\
\hline \hline (Semi-) Manually created & & & & & & \\
ElhPolar & 4673 & .94 & .94 & $\mathbf{. 9 4}$ & .64 & .64 & $\mathbf{. 6 4}$ \\
Mihalcea(f) & 1347 & .91 & .96 & .93 & .61 & .41 & .49 \\
SO-CAL & 4664 & .92 & .96 & .94 & .70 & .51 & .59 \\
\hline
\end{tabular}

Table 6: Evaluation of Spanish lexicons using the full HOpinion corpus at word level.

\subsection{Intrinsic evaluation}

To facilitate intrinsic comparison with previous approaches, we evaluate our automatically generated lexicons against GI. For each qwn-ppv lexicon shown in previous extrinsic evaluations, we compute the intersection between the lexicon and GI, and evaluate the words in that intersection. Table 7 shows results for the best-performing QWNPPV lexicons (both using AG and TL seeds) in the extrinsic evaluations at word level of tables 1 (first two rows), 2 (rows 3 and 4) and 4 (rows 5 and 6). We can see that QWN-PPV lexicons systematically outperform SWN in number of correct entries. $Q W N-P P V-T L$ lexicons obtain $75.04 \%$ of correctness on average. The best performing lexicon contains up to $81.07 \%$ of correct entries. Note that we did not compare the results with MSOL(ASL-GI) because it contains the GI.

\begin{tabular}{lccccc}
\hline Lexicon & $\cap$ wrt. GI & \multicolumn{3}{c}{ Acc. Pos } & Neg \\
& & & & & \\
\hline \hline SWN & 2,755 & .74 & .76 & .73 \\
QWN-PPV-AG (w01_G1) & 849 & .71 & .68 & .75 \\
QWN-PPV-TL (w01_G1) & 713 & .78 & .80 & .76 \\
QWN-PPV-AG (s09_G4) & 3,328 & .75 & .75 & .77 \\
QWN-PPV-TL (s05_G4) & 3,333 & $\mathbf{. 8 0}$ & $\mathbf{. 8 4}$ & $\mathbf{. 7 7}$ \\
QWN-PPV-AG (w02_G3) & 3,340 & .74 & .71 & .77 \\
QWN-PPV-TL (s06_G3) & 3,340 & .77 & .79 & .77 \\
\hline
\end{tabular}

Table 7: Accuracy QWN-PPV lexicons and SWN with respect to the GI lexicon.

\subsection{Discussion}

$Q W N-P P V$ lexicons obtain the best results among the evaluations for English and Spanish. Furthermore, across tasks and datasets $q w n-p p v$ provides a more consistent and robust behaviour than most of the manually-built lexicons apart from OF. The results also show that for a task requiring high 
recall the larger graphs, e.g. G3, are preferable, whereas for a more balanced dataset and document level task smaller G1 graphs perform better.

These are good results considering that our method to generate $q w n-p p v$ is simpler, more robust and adaptable than previous automatic approaches. Furthermore, although also based on a Personalized PageRank application, it is much simpler than SentiWordNet 3.0, consistently outperformed by qwn-ppv on every evaluation and dataset. The main differences with respect to SentiWordNet's approach are the following: (i) the seed generation and training of 7 supervised classifiers corresponds in $q w n-p p v$ to only one simple step, namely, the automatic generation of seeds as explained in section 3.1; (ii) the generation of qwn-ppv only requires a LKB's graph for the Personalized PageRank propagation, no disambiguated glosses; (iii) the graph they use to do the propagation also depends on disambiguated glosses, not readily available for any language.

The fact that qwn-ppv is based on already available WordNets projected onto simple graphs is crucial for the robustness and adaptability of the qwn-ppv method across evaluation tasks and datasets: Our method can quickly create, over different graphs, many lexicons of diffent sizes which can then be evaluated on a particular polarity classification task and dataset. Hence the different configurations of the qwn-ppv lexicons, because for some tasks a G3 graph with more AG/TL seed iterations will obtain better recall and viceversa. This is confirmed by the results: the tasks using MPQA seem to clearly benefit from high recall whereas the Bespalov's corpus has overall, more balanced scores. This could also be due to the size of Bespalov's corpus, almost 10 times larger than MPQA 1.2.

The experiments to generate Spanish lexicons confirm the trend showed by the English evaluations: Lexicons generated by qwn-ppv consistenly outperform other automatic approaches, although some manual lexicon is better on a given task and dataset (usually a different one). Nonetheless the Spanish evaluation shows that our method is also robust across languages as it gets quite close to the manually corrected lexicon of Mihalcea(full) (Pérez-Rosas et al., 2012).

The results also confirm that no single lexicon is the most appropriate for any SA task or dataset and domain. In this sense, the adaptability of qwn-ppv is a desirable feature for lexicons to be employed in SA tasks: the unsupervised qwn-ppv method only relies on the availability of a LKB to build hundreds of polarity lexicons which can then be evaluated on a given task and dataset to choose the best fit. If not annotated evaluation set is available, G3-based propagations provide the best recall whereas the G1-based lexicons are less noisy. Finally, we believe that the results reported here point out to the fact that intrinsic evaluations are not meaningful to judge the adequacy a polarity lexicon for a specific SA task.

\section{Concluding Remarks}

This paper presents an unsupervised dictionarybased method qwn-ppv to automatically generate polarity lexicons. Although simpler than similar automatic approaches, it still obtains better results on the four extrinsic evaluations presented. Because it only depends on the availability of a LKB, we believe that this method can be valuable to generate on-demand polarity lexicons for a given language when not sufficient annotated data is available. We demonstrate the adaptability of our approach by producing good performance polarity lexicons for different evaluation scenarios and for more than one language.

Further work includes investigating different graph projections of WordNet relations to do the propagation as well as exploiting synset weights. We also plan to investigate the use of annotated corpora to generate lexicons at word level to try and close the gap with those that have been (at least partially) manually annotated.

The qwn-ppv lexicons and graphs used in this paper are publicly available (under CC-BY license): http://adimen.si.ehu.es/web/qwn-ppv. The $q w n-p p v$ tool to automatically generate polarity lexicons given a WordNet in any language will soon be available in the aforementioned URL.

\section{Acknowledgements}

This work has been supported by the OpeNER FP7 project under Grant No. 296451, the FP7 NewsReader project, Grant No. 316404 and by the Spanish MICINN project SKATER under Grant No. TIN2012-38584-C06-01. 


\section{References}

R. Agerri and A. García-Serrano. 2010. Q-WordNet: extracting polarity from WordNet senses. In Seventh Conference on International Language Resources and Evaluation, Malta. Retrieved May, volume 25, page 2010 .

Eneko Agirre and Aitor Soroa. 2009. Personalizing pagerank for word sense disambiguation. In Proceedings of the 12th Conference of the European Chapter of the Association for Computational Linguistics (EACL-2009), Athens, Greece.

Aitor González Agirre, Egoitz Laparra, German Rigau, and Basque Country Donostia. 2012. Multilingual central repository version 3.0: upgrading a very large lexical knowledge base. In GWC 2012 6th International Global Wordnet Conference, page 118.

Eneko Agirre, Oier Lopez de Lacalle, and Aitor Soroa. 2014. Random walks for knowledge-based word sense disambiguation. Computational Linguistics, (Early Access).

S. Baccianella, A. Esuli, and F. Sebastiani. 2010. SentiWordNet 3.0: An enhanced lexical resource for sentiment analysis and opinion mining. In Seventh conference on International Language Resources and Evaluation (LREC-2010), Malta., volume 25.

Dmitriy Bespalov, Bing Bai, Yanjun Qi, and Ali Shokoufandeh. 2011. Sentiment classification based on supervised latent n-gram analysis. In Proceedings of the 20th ACM international conference on Information and knowledge management, pages 375-382.

Francis Bond and Ryan Foster. 2013. Linking and extending an open multilingual wordnet. In 51st $\mathrm{An}$ nual Meeting of the Association for Computational Linguistics: ACL-2013.

Sergey Brin and Lawrence Page. 1998. The anatomy of a large-scale hypertextual web search engine. Computer networks and ISDN systems, 30(1): 107117 .

Y. Choi and C. Cardie. 2009. Adapting a polarity lexicon using integer linear programming for domainspecific sentiment classification. In Proceedings of the 2009 Conference on Empirical Methods in Natural Language Processing: Volume 2-Volume 2, pages 590-598.

X. Ding, B. Liu, and P. S. Yu. 2008. A holistic lexiconbased approach to opinion mining. In Proceedings of the international conference on Web search and web data mining, pages 231-240.

Andrea Esuli and Fabrizio Sebastiani. 2007. Pageranking wordnet synsets: An application to opinion mining. In Proceedings of the 45th Annual Meeting of the Association of Computational Linguistics, pages 424-431, Prague, Czech Republic, June. Association for Computational Linguistics.
C. Fellbaum and G. Miller, editors. 1998. Wordnet: An Electronic Lexical Database. MIT Press, Cambridge (MA).

V. Hatzivassiloglou and K. R McKeown. 1997. Predicting the semantic orientation of adjectives. In Proceedings of the eighth conference on European chapter of the Association for Computational Linguistics, pages 174-181.

M. Hu and B. Liu. 2004. Mining and summarizing customer reviews. In Proceedings of the tenth ACM SIGKDD international conference on Knowledge discovery and data mining, pages 168-177.

Soo-Min Kim and Eduard Hovy. 2004. Determining the sentiment of opinions. In Proceedings of Coling 2004, pages 1367-1373, Geneva, Switzerland, Aug 23-Aug 27. COLING.

Bing Liu. 2012. Sentiment analysis and opinion mining. Synthesis Lectures on Human Language Technologies, 5(1):1-167.

R. Mihalcea, C. Banea, and J. Wiebe. 2007. Learning multilingual subjective language via cross-lingual projections. In Annual Meeting of the Association for Computational Linguistics, volume 45, page 976.

S. Mohammad, C. Dunne, and B. Dorr. 2009. Generating high-coverage semantic orientation lexicons from overtly marked words and a thesaurus. In Proceedings of the 2009 Conference on Empirical Methods in Natural Language Processing: Volume 2-Volume 2, pages 599-608.

Lluís Padró and Evgeny Stanilovsky. 2012. Freeling 3.0: Towards wider multilinguality. In Proceedings of the Language Resources and Evaluation Conference (LREC 2012), Istanbul, Turkey, May. ELRA.

B. Pang and L. Lee. 2008. Opinion mining and sentiment analysis. Foundations and Trends in Information Retrieval, 2(1-2):1-135.

Verónica Pérez-Rosas, Carmen Banea, and Rada Mihalcea. 2012. Learning sentiment lexicons in spanish. In LREC, pages 3077-3081.

D. Rao and D. Ravichandran. 2009. Semi-supervised polarity lexicon induction. In Proceedings of the 12th Conference of the European Chapter of the Association for Computational Linguistics, pages 675682.

E. Riloff and J. Wiebe. 2003. Learning extraction patterns for subjective expressions. In Proceedings of the International Conference on Empirical Methods in Natural Language Processing (EMNLP'03).

Xabier Saralegi and Iñaki San Vicente. 2013. Elhuyar at TASS2013. In XXIX Congreso de la Sociedad Espaola de Procesamiento de lenguaje natural, Workshop on Sentiment Analysis at SEPLN (TASS2013), pages 143-150, Madrid. 
P. Stone, D. Dunphy, M. Smith, and D. Ogilvie. 1966. The General Inquirer: A Computer Approach to Content Analysis. Cambridge (MA): MIT Press.

Carlo Strapparava and Alessandro Valitutti. 2004. Wordnet-affect: an affective extension of wordnet. In Proceedings of the 4th International Conference on Languages Resources and Evaluation (LREC 2004), pages 1083-1086, Lisbon, May.

M. Taboada, J. Brooke, M. Tofiloski, K. Voll, and M. Stede. 2010. Lexicon-based methods for sentiment analysis. Computational Linguistics, (Early Access):141.

Hiroya Takamura, Takashi Inui, and Manabu Okumura. 2005. Extracting semantic orientations of words using spin model. In Proceedings of the 43rd Annual Meeting of the Association for Computational Linguistics (ACL'05), page 133140, Ann Arbor, Michigan, June. Association for Computational Linguistics.

P. Turney and M. Littman. 2003. Measuring praise and criticism: Inference of semantic oreintation from association. ACM Transaction on Information Systems, 21(4):315-346.

P.D. Turney. 2002. Thumbs up or thumbs down?: semantic orientation applied to unsupervised classification of reviews. In Proceedings of the 40th Annual Meeting on Association for Computational Linguistics, page 417424.

Theresa Wilson, Janyce Wiebe, and Paul Hoffmann. 2005. Recognizing contextual polarity in phraselevel sentiment analysis. In Proceedings of the conference on Human Language Technology and Empirical Methods in Natural Language Processing, page 347354. 